\title{
Microscopic model of surfaces in orientationally disordered ionic crystals: The (001) surface of $\mathrm{KCN}$
}

\author{
P. Zieliński* \\ Institut für Experimentalphysik der Universität Wien, Strudlhofgasse 4, A-1090 Wien, Austria \\ K. H. Michel \\ Department Natuurkunde, Universitaire Instelling Antwerpen, Universiteitsplein 1, B-2610 Wilrijk, Belgium
}

(Received 4 February 1992)

\begin{abstract}
The crystallographic structure and the distribution of orientations of molecular ions are studied near the surface in an orientationally disordered crystal with the use of a Green-function formalism. The orientational degrees of freedom are treated by means of symmetry-adapted functions of angular coordinates. The structure of the (001) surface of $\mathrm{KCN}$ in its cubic fcc phase is then predicted using the existing data on the interaction of the ions $\mathrm{K}^{+}$and $\mathrm{CN}^{-}$. A local antiferroelectric and antiferroelastic order is shown to exist in the surface region. The magnitude of the order and the spatial extent of the ordered region increase as the temperature approaches the point of the phase transition to the ordered phase. The influence of the external electric field on the structure of the surface is predicted.
\end{abstract}

\section{INTRODUCTION}

The structure and the dynamics of crystal surfaces have been subject recently to numerous experimental and theoretical studies. In particular, the diffraction techniques: low-energy electron diffraction, helium diffraction, neutron and x-ray scattering at grazing angles, as well as the recently developed tunneling microscopy techniques allow for a rather exact determination of the arrangement of atoms in the surface regions. ${ }^{1}$

The main interest in the structure and the dynamics of surface now concerns the metallic, semiconducting, and simple ionic materials first because of their relative facility to prepare well-characterized surfaces and, second, because of their technological applications. Among the materials of more complex structure the ferroelectrics turn out to be of the greatest interest with respect to surface effects. It has been established that the surfaces play an important role in the onset of the spontaneous polarization, ${ }^{2}$ especially when the crystal has the form of a thin layer. Only few works have appeared on the surfaces in crystals possessing orientational degrees of freedom ${ }^{3}$ and the majority of them concern the orientationally ordered phases.

It is generally accepted that the surfaces of ionic crystals are smooth and the surfaces of orientationally disordered crystals built up of neutral molecules called molecular crystals are rough. It is, therefore, interesting to study surfaces in ionic crystals, in which at least the ions of one kind are polyatomic and show an orientational disorder in some range of temperatures. The tendencies to the smoothness and to the roughness are then expected to influence the real structure of such surfaces.

In the present paper a method of the theoretical treatment of surfaces in ionic crystals exhibiting an orientational disorder of one type of the ions, practically almost always the anions, is developed under the assumption that the surface is smooth from the point of view of translational degrees of freedom. This means that the ions, no matter whether monatomic or orientationally disordered polyatomic, are supposed to occupy welldefined sites at all the crystallographic planes, the topmost plane of the crystal with surface included. Thus, the effect of the surface on the structure of the system can be described in terms of the translational displacements of the ions' centers of mass with respect to their positions peculiar to the bulk crystal and in terms of the distortions of the distribution of the orientational probability at the sites occupied by the polyatomic ions. The physical reason for the displacements and the distortions to occur lies in the geometry of the crystal with surface. Indeed, all the forces exerted on a given ion by the other ions must sum to zero in the infinite crystal in equilibrium. Near to the surface, however, the forces become unbalanced due to the lack of the ions beyond the surface plane. Analogously, the lack of the ions beyond the surface modifies the local orientational potential acting on the polyatomic ions compared to the potential in the bulk. The modification of the potential is a thermodynamical force conjugate to the distribution of orientations. All the mentioned kinds of forces can be calculated from the microscopic interatomic potentials. Within a harmonic or a pseudoharmonic approximation the structure of the crystal-with-surface is defined by the linear reaction of the truncated crystal to the above forces. The structure is then called relaxed and the phenomenon surface relaxation. ${ }^{1}$ When the forces are very strong, nonlinear or anharmonic reaction has to be taken into account, which can in some cases produce a reconstruction of the surface. ${ }^{1}$ The equilibrium structure of the topmost lattice plane is then different from that of the crystallographic planes in the bulk, e.g., it has a different spatial periodicity. In the present work we calculate the expected surface relaxation of the translational and orientation- 
al degrees of freedom within a pseudoharmonic approximation.

There exist several kinds of theoretical treatment of the structure and dynamics of solid surfaces. In the slabcalculation method ${ }^{3}$ one finds the equilibrium configuration of atoms in a model crystal consisting of a finite number of atomic planes parallel to the surface under question. The vibrational frequencies of such a layer are then determined by the diagonalization of the corresponding dynamical matrix which is a function of the wave vector parallel to the surface and whose dimension depends on the thickness of the slab. This technique is especially well adapted to the cases where the surface effects do not penetrate very deep into the crystal. In contrast to that, the Green-function technique allows one to treat semi-infinite crystals and it is usually a question of approximation how deep perturbations are taken into account. $^{4}$ In both above-mentioned techniques the thermal effects are accounted for by some statistical averaging or, more often, by some phenomenological temperature dependence of the interaction parameters. Computer simulations provide a more realistic description of the atomic motions near to the surface. ${ }^{5}$

In the present paper the Green-function technique is employed to predict equilibrium structure of the orientationally disordered ionic crystals with surfaces. The choice of the method stems from the fact that these crystals usually undergo a disorder-order phase transition and, therefore, the surface effects are supposed to extend deep into the bulk at temperatures close to the ordering temperature. Formally, the Green function is in our case a semi-infinite matrix inverse to the matrix, which defines the quadratic form of the Landau free energy of the crystal with the surface expressed as a function of the variables of interest, i.e., the translational displacements and the distortions of the orientational distribution. Therefore, the Green function defines the linear reaction of the semi-infinite crystal to the external forces, also the mentioned forces related with the truncation of the crystal. The elements of the Green-function matrix are calculated here on the basis of the surface and interface response theory due to Dobrzynski ${ }^{6}$ with some modifications for long-range interactions. ${ }^{7,8}$

As a real example we select the orientationally disordered cubic phase of $\mathrm{KCN}$ since the microscopic interactions and the local properties of the orientational disorder are already well established in the bulk crystal..$^{9-11}$ The $\mathrm{CN}^{-}$ion is of particular interest since it possesses at the same time an electric dipole and a quadrupole moment.

In Sec. II we present the construction of the freeenergy matrix for the semi-infinite crystal using microscopic quantities and their bulk averages. The most important element of the theory is the replacement of the angular variables of the reorienting molecules by corresponding symmetry-adapted multipole functions. ${ }^{12}$ This has been shown to make the problem of large angle rotational motions tractable. The predicted structure is then obtained with the use of the corresponding Green function. The specific form of the free-energy matrix for the (001) surface of $\mathrm{KCN}$ is given in Sec. III. Advantage is taken of the symmetry of the problem to reduce the number of coefficients. The numerical values of the coefficients are obtained using the existing experimental and theoretical data on the interactions of the ions $\mathrm{K}^{+}$ and $\mathrm{CN}^{-}$. The corresponding profiles of translational displacements and the distortions of the orientational probability distribution near to the (001) surface of KCN are given in Sec. IV. The structure turns out locally antiferroelectric with the polarization vector parallel to the surface normal and antiferroelastic. The influence of the external electric field parallel and perpendicular to the surface is also presented. The range of applicability and the possible differences between the predictions of the present theory and the real structure are discussed in Sec. V.

\section{GREEN-FUNCTION DESCRIPTION OF THE SURFACES IN ORIENTATIONALLY DISORDERED CRYSTALS}

Any crystal with a surface cleaved in a given crystallographic orientation can be regarded as a semi-infinite succession of mutually parallel atomic or ionic lattice planes. In the bulk or far enough from the surface the lattice planes are identical and equidistant so that the threedimensional spatial periodicity of the crystal is ensured. The truncation of the series of the lattice planes at the surface lifts the periodicity in the direction perpendicular to the surface. At the same time, the forces acting on the individual atoms, which all vanish in the equilibrium state of the bulk material, become noncompensated in the vicinity of the surface. As a result, the mutual distances of the lattice planes undergo a variation, usually called surface relaxation. For the same reason the arrangement of the atoms or ions within the planes close to the surface also change giving rise to so-called surface reconstruction. The extent of the phenomena depends on the magnitude of the forces with respect to the restoring forces tending to enhance the bulk structure. Thanks to the diperiodicity of the system in the directions parallel to the surface all the variations of the structure can be characterized by the two-dimensional wave vector $\mathbf{k}_{\|}$from the corresponding two-dimensional Brillouin zone. In what follows we shall concentrate on the variations of the structure corresponding to $\mathbf{k}_{\|}=0$, first because the forces arising at the (001) surface of the $\mathrm{KCN}$ crystal taken as the example are also characterized by $k=0$ and, secondly, because the Green-function technique for this wave vector requires a special treatment of variables.

\section{A. The variables}

Let the lattice planes making up the crystal with surface consist of cations and anions. [In the rocksalt structure of $\mathrm{KCN}$ this is the case for the (001) surface and not, e.g., for the (111) surface).] At $\mathbf{k}_{\|}=0$ the displacements of cations $\mathbf{u}^{+}(l)$ and anions $\mathbf{u}^{-}(l)$ are identical at all the equivalent sites belonging to the same lattice plane $l$. Since a translation of all the ions by the same vector costs no energy, every Hamiltonian involving the variables $\mathbf{u}^{+}(l)$ and $\mathbf{u}^{-}(l)$ has one vanishing eigenvalue and there- 
fore the corresponding Green function does not exist. ${ }^{7}$ To avoid this difficulty it is convenient to introduce the new variables

$$
\mathbf{x}(l)=\frac{1}{2}\left[\mathbf{u}^{+}(l+1)+\mathbf{u}^{-}(l+1)-\mathbf{u}^{+}(l)-\mathbf{u}^{-}(l)\right]
$$

and

$$
\mathbf{y}(l)=\frac{1}{2}\left[\mathbf{u}^{+}(l)-\mathbf{u}^{-}(l)\right] .
$$

The variable $\mathbf{x}(l)$ corresponds to the local strain. In case of the $z$ axis perpendicular to the surface one has $x_{1}(l)=\epsilon_{13}(l) a, x_{2}(l)=\epsilon_{23}(l) a$, and $x_{3}(l)=\epsilon_{33}(l) a$, where $a$ is the distance between the lattice planes in the bulk. When the masses of anions and cations are equal the Fourier transform of the variables $\mathbf{y}(l)$ are directly equal to the displacements involved in the optical phonons propagating perpendicular to the surface.

The rotational degrees of freedom in the orientationally disordered crystals are accounted for with the use of the symmetry-adapted functions. ${ }^{11-13}$ The orientational disorder is then characterized by the single-particle angular distribution $P_{0}(\Omega)$,

$$
P_{0}(\Omega)=Z_{0}^{-1} \exp \left[-\beta W_{0}^{R}(\Omega)\right],
$$

where

$$
\begin{aligned}
& Z_{0} \int \exp \left[-\beta W_{0}^{R}(\Omega)\right] d \Omega, \\
& \beta=1 / T .
\end{aligned}
$$

$\Omega$ stands for the polar angles $(\theta, \phi)$ which specify the orientation of the linear $\mathrm{CN}^{-}$ion. $W_{0}^{R}(\Omega)$ is the effective, single-particle potential which in the bulk material possesses the full symmetry of the site point group. When an external field $\mathbf{E}_{\lambda}$ is applied so that the symmetry of the site is broken the local potential changes,

$$
W^{R}(\Omega)=W_{0}^{R}(\Omega)-\sum_{\lambda, \mu} E_{\lambda}^{\mu} Y_{\lambda}^{\mu}(\Omega)
$$

and, consequently, the single-particle orientational distribution becomes to the first order in $E_{\lambda}^{\mu}$,

$$
P(\Omega)=P_{0}(\Omega)\left(1+\beta \sum_{\lambda, \mu} E_{\lambda}^{\mu} Y_{\lambda}^{\mu}(\Omega)\right) .
$$

Here $Y_{\lambda}^{\mu}(\Omega)$ denote linear combinations of spherical harmonics which have the full symmetry of the reorienting molecule but a lower symmetry than is required by the site point group in the bulk of the crystal. In the case of $\mathrm{CN}^{-}$molecular ions the field $E_{1}^{\mu}(\mu=1,2,3)$ stands for any interaction enhancing a head tail order, i.e., it is a field conjugate to the dipolar orientational distribution. It encompasses the local electric field but also some short-range forces which distinguish both ends of the anion. Similarly, the field $E_{2}^{\mu}(\mu=1, \ldots, 5)$ enhances a quadrupolar ordering of the anions. It will be shown that the truncation of the $\mathrm{KCN}$ crystal produces both kinds of fields.

When considering the structural distortions caused by the surface and corresponding to $\mathbf{k}_{\|}=0$ one assumes that the modification of the orientational distribution $Y_{\lambda}^{\mu}(\Omega)$ is identical at all the equivalent sites belonging to the same lattice plane $l$ and depends on the label $l$ only: $Y_{\lambda}^{\mu}(\Omega(l))=Y_{\lambda}^{\mu}(l)$. Taking into account all the abovedefined variables and assuming that only the anions have orientational degrees of freedom of the dipolar and quadrupolar type, one can characterize the state of the crystal by the following 14-component vector $\mathrm{w}(l)$ of generalized displacements ascribed to every lattice plane $l=1, \ldots, \infty$,

$$
\mathbf{w}(l)=(\mathbf{x}(l), \mathbf{y}(l), \mathbf{P}(l), \mathbf{Y}(l)) \text {. }
$$

One should remark that the displacement components $\mathbf{x}(l)$ and $\mathbf{y}(l)$ have the dimension of length, while the orientational variables $P(l)$ and $\mathrm{Y}(l)$ are dimensionless.

To avoid too many indices we have defined

$$
P_{i}(l)=Y_{1, i}^{u}(l), \quad i=1, \ldots, 3
$$

which specify the dipolar orientations,

$$
Y_{i}(l)=Y_{2, i}^{e}(l), \quad i=1,2
$$

which specify the quadrupolar orientation of the $E_{g}$ symmetry, and

$$
Y_{i}(l)=Y_{2, i-2}^{f}(l), \quad i=3, \ldots, 5
$$

which specify the quadrupolar orientations of the $T_{2 g}$ symmetry. The symmetry-adapted harmonics $Y_{l, i}^{\alpha}$, $\alpha=u, e, f$ are explicitly given in Ref. 11. In Cartesian coordinates, these functions read

$$
\begin{aligned}
& Y_{1,1}^{u}=(3 / 4 \pi)^{1 / 2} x, \\
& Y_{1,2}^{u}=(3 / 4 \pi)^{1 / 2} y, \\
& Y_{1,3}^{u}=(3 / 4 \pi)^{1 / 2} z, \\
& Y_{2,1}^{e}=(5 / 16 \pi)^{1 / 2}\left(3 z^{2}-1\right), \\
& Y_{2,2}^{e}=(15 / 16 \pi)^{1 / 2}\left(x^{2}-y^{2}\right), \\
& Y_{2,1}^{f}=(15 / 4 \pi)^{1 / 2} x y, \\
& Y_{2,2}^{f}=(15 / 4 \pi)^{1 / 2} x z, \\
& Y_{2,3}^{f}=(15 / 4 \pi)^{1 / 2} y z,
\end{aligned}
$$

where $x=\sin \theta \cos \phi, y=\sin \theta \sin \phi, z=\cos \phi$.

\section{B. The equilibrium structure of the crystal with surface}

The free energy per two-dimensional unit cell of the crystal with surface can be generally written as follows:

$$
\begin{aligned}
F(\{\mathbf{w}(l)\}, T)= & F_{0}(T)+\frac{1}{2} \sum_{l, l^{\prime}=1}^{\infty}\left\langle\mathbf{w}^{\dagger}(l)\right\rangle \overleftrightarrow{\mathrm{h}}\left(l, l^{\prime} ; T\right)\left\langle\mathbf{w}\left(l^{\prime}\right)\right\rangle \\
& -\sum_{l=1}^{\infty}\left\langle\mathbf{w}^{\dagger}(l)\right\rangle \mathbf{f}(l)
\end{aligned}
$$

where $\langle\mathbf{w}(l)\rangle$ are the thermal averages of the generalized displacement vector $\mathbf{w}(l)$ [Eq. (6)]. $\overleftrightarrow{\mathrm{h}}\left(l, l^{\prime} ; T\right)$ is a semiinfinite temperature-dependent matrix whose elements will be defined in Sec. II for the KCN (001) surface. $\mathbf{f}(l)$ are the forces arising as the result of the truncation of the crystal or the external forces applied to the system. The 
free energy [Eq. (8)] is expanded to the second order in $\mathbf{w}(l)$ only and does not account for nonlinear phenomena.

The structure of the crystal with surface is defined by the minimum of the free energy with respect to the variables $\mathbf{w}(l)$,

$$
\frac{\partial F}{\partial \mathrm{w}(l)}=\sum_{l^{\prime}=1}^{\infty} \overleftrightarrow{\mathrm{h}}\left(l, 1^{\prime}\right) \mathbf{w}(l)-\mathbf{f}(l)=0 .
$$

The formal solution of the system of equations [Eq. (9)]

$$
\mathbf{w}(l)=\sum_{l^{\prime}=1}^{\infty} \overleftrightarrow{\mathrm{g}}\left(l, l^{\prime} ; T\right) \mathbf{f}\left(l^{\prime}\right)
$$

involves the static Green function $\overleftrightarrow{\mathrm{g}}\left(l^{\prime}, l^{\prime} ; T\right)$ defined by

$$
\sum_{l^{\prime \prime}=1}^{\infty} \overleftrightarrow{\mathrm{g}}\left(l, l^{\prime \prime} ; T\right) \overleftrightarrow{\mathrm{h}}\left(l^{\prime \prime}, l^{\prime} ; T\right)=\overleftrightarrow{\mathrm{I}} \delta_{l, l^{\prime}},
$$

where $\overleftrightarrow{\mathrm{I}}$ is a $14 \times 14$ unit matrix.

The scheme of the determination of the surface structure presented in this section is a discrete version of the continuum theory due to Kroll and Lipowsky ${ }^{14}$ and Tilley and Žekš. ${ }^{15}$ In those theories the free-energy matrix $\overleftrightarrow{\mathrm{h}}\left(l^{\prime} l^{\prime} ; T\right)$ is represented by a squared gradient term $(\nabla \eta)^{2}$ of the order parameter $\eta$. Such an approximation would correspond in the present case to a one-component vector $\mathbf{w}(l)=\eta(l)$ and to only nearest-neighbor interactions. The differential equations of the continuum theory are now replaced by the difference equations [Eq. (9)].

\section{THE FREE ENERGY FOR THE (001) SURFACE OF THE CUBIC KCN CRYSTAL}

\section{A. Symmetry considerations}

By cleaving a rocksalt crystal perpendicular to the (001) direction one obtains a system of the symmetry of the two-dimensional space group $p 4 \mathrm{~mm} .{ }^{16}$ At $\mathbf{k}_{\|}=0$ all the changes of the structure expressed by nonzero values of the variables given in Eq. (6) can be classified according to the irreducible representations of the point group $4 \mathrm{~mm}$.

The translational displacements $x_{3}(l), y_{3}(l)$, the polarization $P_{3}(l)$, and the quadrupole variable $Y_{1}(l)$ show the full symmetry $4 \mathrm{~mm}$ and thus transform according to the representation $A_{1}$. The transverse translations $x_{i}(l)$, $y_{i}(l)$, and the polarization $P_{i}(l)$ for $i=1,2$ as well as the quadrupole distortions $Y_{5}$ and $Y_{4}$ (in this order) constitute the basis functions of the two-dimensional representation $E$. The remaining symmetry-adapted harmonics $Y_{2}$ and $Y_{3}$ belong to the representations $B_{1}$ and $B_{2}$, respectively. From this symmetry analysis it follows that the $14 \times 14$ matrices $\overleftrightarrow{\mathrm{h}}\left(l, l^{\prime} ; T\right)$ decouple into a blockdiagonal form, every block corresponding to one irreducible representation. Moreover, the block of the symmetry $E$ also consists of two decoupled identical $4 \times 4$ blocks corresponding to every basis function of this representation.

The forces arising as a result of the truncation of the ideal (001) surface in this structure have all the full symmetry of the group $4 \mathrm{~mm}$, i.e., correspond to the represen- tation $A_{1}$. This means that without external fields the vector $\mathbf{f}(l)$ of Eq. (8) has only nonvanishing components conjugated to the variables $x_{3}(l), y_{3}(l), P_{3}(l)$, and $Y_{1}(l)$. Qualitatively, therefore, the clean surface $(001)$ of $\mathrm{KCN}$ and of all isostructural materials can show a longitudinal relaxation expressed by $x_{3}(l)$, a $1 \times 1$ reconstruction $y_{3}(l)$ consisting of a mutual shift of the cations and anions perpendicular to the surface, a perpendicular orientational polarization $P_{3}(l)$ and, finally, a variation of the orientational distribution of the quadrupoles. The latter variation has a cylindrical symmetry with respect to the $z$ axis.

An external electric field perpendicular to the surface will produce an $l$-independent force acting on $y_{3}(l)$ and $P_{3}(l)$. An electric field parallel to the surface will analogously produce an $l$-independent force acting on $y_{1}(l)$, $y_{2}(l), P_{1}(l)$, and $P_{2}(l)$, thus revealing the features of the corresponding Green function [Eq. (11)].

The magnitude of the above-mentioned distortions is determined by the numerical values of the elements of the matrix $\overleftrightarrow{\mathrm{h}}\left(l, l^{\prime} ; T\right)$ and of the forces $\mathbf{f}(l)$.

\section{B. Numerical values of the free-energy coefficients}

The microscopic parameters of the interionic interactions in the KCN bulk crystal have been obtained in Ref. 11. They can be immediately related to the coefficients of the matrix $\overleftrightarrow{\mathrm{h}}\left(l, l^{\prime} ; T\right)$.

The $4 \times 4$ block of the $A_{1}$ symmetry has the following general form:

$$
\begin{aligned}
& \overleftrightarrow{\mathrm{h}}_{A}(l, l ; T)=\left[\begin{array}{cccc}
\alpha_{0}^{A} & 0 & -D & 2 A \\
0 & \omega_{0}^{A} & -R & 0 \\
-D & -R & \delta_{0}^{A} & 0 \\
2 A & 0 & 0 & J_{0}^{A}
\end{array}\right], \\
& \overleftrightarrow{\mathrm{h}}_{A}(l, l+1 ; T)=\left[\begin{array}{cccc}
\alpha_{1}^{A} & 0 & D & 2 A \\
0 & \omega_{1}^{A} & -D & -2 A \\
0 & -D & \delta_{1}^{A} & 0 \\
0 & 2 A & 0 & J_{1}^{A}
\end{array}\right], \\
& \overleftrightarrow{\mathrm{h}}_{A}(l, l-1 ; T)=\stackrel{\mathrm{h}_{A}^{T}(l, l+1 ; T),}{ }
\end{aligned}
$$

where $R=2 D+4 E$. All the other elements of the matrix $\overleftrightarrow{\mathrm{h}}_{A}\left(l, l^{\prime} ; T\right)$ vanish.

The $4 \times 4$ block corresponding to the transverse variables $x_{1}(l), y_{1}(l), P_{1}(l)$, and $Y_{5}(l)$ has a similar form,

$$
\begin{aligned}
& \overleftrightarrow{\mathrm{h}}_{E}(l, l ; T)=\left[\begin{array}{cccc}
\alpha_{0}^{E} & 0 & -E & -B \\
0 & \omega_{0}^{E} & -R & 0 \\
-E & -R & \delta_{0}^{E} & 0 \\
-B & 0 & 0 & J_{0}^{E}
\end{array}\right], \\
& \overleftrightarrow{\mathrm{h}}_{E}(l, l+1 ; T)=\left[\begin{array}{cccc}
\alpha_{1}^{E} & 0 & E & -B \\
0 & \omega_{1}^{E} & -E & B \\
0 & -E & \delta_{1}^{E} & 0 \\
0 & -B & 0 & J_{1}^{E}
\end{array}\right],
\end{aligned}
$$




$$
\overleftrightarrow{\mathrm{h}}_{E}(l, l-1 ; T)=\overleftrightarrow{\mathrm{h}}_{E}^{T}(l, l+1 ; T)
$$

Finally

$$
h_{\lambda}\left(l, l^{\prime} ; T\right)=J_{0}^{\lambda} \delta_{l l^{\prime}}+J_{1}^{\lambda}\left(\delta_{l l^{\prime}+1}+\delta_{l l^{\prime}-1}\right)
$$

for $\lambda=B_{1}, B_{2}$.

Following the convention applied in Refs. 10 and 11 we express the free energy per two-dimensional unit cell in units of $\mathrm{K}$. Correspondingly in Eqs. (12)-(14), the coefficients $\alpha_{i}^{\lambda}$ and $\omega_{i}^{\lambda}$ have the units $\mathrm{K} / \AA^{2}$, the coefficients $\delta_{i}^{\lambda}$ and $J_{i}^{\lambda}$ have the units $K$, and the coefficients $A, R, B, D$, and $E$ the units $\mathrm{K} / \AA$.

The rotation-translation coupling coefficients $A, B, D$, and $E$ have been evaluated in Ref. 11. The parameters $\alpha_{i}^{A, E}, \omega_{i}^{A, E}, i=1,2$ characterize the bare translational interactions. In the present calculations they have been extracted from the "bare" dispersion relations fitted to the experimental phonon frequencies by Strauch, Schröder, and Bauerfeind. ${ }^{17}$ To achieve this extraction the following reasoning has been applied. The bare dispersion curves result from an interplay between the masses of the $\mathrm{K}^{+}$and $\mathrm{CN}^{-}$ions and some effective force constants encompassing the polarizability of the ions. However, the shell-model calculations of Strauch, Schröder, and Bauerfeind contains all kinds of polarizability, the orientational polarizability of the disordered polar anions $\mathrm{CN}^{-}$included, whereas in the present work the orientational interactions of the molecular permanent dipoles are treated separately. Consequently, the force constants $\alpha_{0}^{A, E}, \alpha_{1}^{A, E}$, $\omega_{0}^{A, E}, \omega_{1}^{A, E}$ used here were deduced under the assumption that the "bare" phonon frequencies given by Strauch, Schröder, and Bauerfeind result from the coupling of the really bare phonons with the dipoles whose interaction is given by $\delta_{0}^{A, E}$ and $\delta_{1}^{A, E}$ and whose coupling to the phonon coordinates is determined by the coefficients $D$ and $E$. This procedure has shown that $\alpha_{1}^{E}$ and $\omega_{1}^{E}$ are $\alpha_{1}^{A}$ are negligibly small compared to $\alpha_{0}^{E}, \omega_{0}^{E}$, and $\alpha_{0}^{A}$ (see Table I), which means that the transverse- and the longitudinalacoustic phonons show practically no spatial dispersion.
The diagonal terms of the purely orientational part of the free-energy matrix contribute to the entropy of the crystal and are temperature-dependent. ${ }^{13}$ One has

$$
\begin{aligned}
& J_{0}^{\lambda}(l, l, T)=\bar{J}_{0}^{\lambda}+\frac{T}{\left\langle\left[Y_{\lambda}(l)\right]^{2}\right\rangle}+C_{Y_{\lambda}}^{s}(l), \\
& \delta_{0}^{\lambda}(l, l, T)=\bar{\delta}_{0}^{\lambda}+\frac{T}{\left\langle\left[P_{\lambda}(l)\right]^{2}\right\rangle}+C_{P_{\lambda}}^{s}(l),
\end{aligned}
$$

where $\bar{J}_{0}^{\lambda}$ and $\bar{\delta}_{0}^{\lambda}$ are the contributions to the direct quadrupole-quadrupole and dipole-dipole interactions coming from the anions located in the same lattice plane. In the bulk the self-interaction terms $C_{Y_{\lambda}}^{s}$ and $C_{P_{\lambda}}^{s}$ are equal for every $l$ and for every symmetry-adapted function of a given rank and belonging to the same irreducible representation of the site group $m 3 m$ of the position occupied by the anion. When this symmetry is lifted by the truncation of the surface the self-interaction terms become $l$ dependent and are equal for the symmetryadapted functions belonging to the same irreducible representation of the group $4 \mathrm{~mm}$. Consequently, in our case the self-interactions have different values for $\left(P_{1}, P_{2}\right), P_{3}$, $Y_{1}, Y_{2}, Y_{3},\left(Y_{5}, Y_{4}\right)$. These dependencies have been indicated in formulas (15) and (16). The same symmetry properties pertain to the one-particle susceptibilities $\left\langle\left[Y_{i}(l)\right]^{2}\right\rangle$ and $\left\langle\left[P_{i}(l)\right]^{2}\right\rangle$, which are the corresponding thermal averages calculated with the lattice field with subtracted self-interaction terms (see Ref. 13 for a detailed discussion).

The terms $J_{1}^{\lambda}$ and $\delta_{1}^{\lambda}$ are the direct quadrupolequadrupole and dipole-dipole interactions of the anions belonging to the neighboring lattice planes. In the present work only nearest-neighbor quadrupolequadrupole interactions have been taken into account,

\begin{tabular}{|c|c|c|c|c|c|}
\hline $\begin{array}{c}\alpha_{0}^{A} \\
\left(\mathrm{~K} / \AA^{2}\right)\end{array}$ & $\begin{array}{c}\alpha_{1}^{A} \\
\left(\mathrm{~K} / \AA^{2}\right)\end{array}$ & $\begin{array}{c}\omega_{0}^{A} \\
\left(\mathrm{~K} / \AA^{2}\right)\end{array}$ & $\begin{array}{c}\omega_{1}^{A} \\
\left(\mathbf{K} / \AA^{2}\right)\end{array}$ & $\begin{array}{c}\alpha_{0}^{E} \\
\left(\mathbf{K} / \AA^{2}\right)\end{array}$ & $\begin{array}{c}\alpha_{1}^{E} \\
\left(\mathbf{K} / \AA^{2}\right)\end{array}$ \\
\hline 21054.7 & -927.4 & 98231.4 & 33708.5 & 2349.3 & -246.6 \\
\hline $\begin{array}{c}\omega_{0}^{E} \\
\left(\mathrm{~K} / \AA^{2}\right)\end{array}$ & $\begin{array}{c}\omega_{1}^{E} \\
\left(\mathbf{K} / \AA^{2}\right)\end{array}$ & $\begin{array}{c}\bar{\delta}_{0}^{A} \\
(\mathbf{K})\end{array}$ & $\begin{array}{c}\delta_{1}^{A} \\
(\mathbf{K})\end{array}$ & $\begin{array}{c}\bar{\delta}_{0}^{E} \\
(\mathbf{K})\end{array}$ & $\begin{array}{c}\delta_{1}^{E} \\
(\mathrm{~K})\end{array}$ \\
\hline 44563.5 & -1861.6 & 782.7 & -62.1 & -539.0 & -31.0 \\
\hline $\begin{array}{c}Q^{2} / a^{5} \\
(\mathbf{K})\end{array}$ & $\begin{array}{c}C_{Y_{1} Y_{2}}^{s} \\
(\mathbf{K})\end{array}$ & $\begin{array}{c}C_{Y_{1} Y_{2} Y_{3}}^{s} \\
(\mathbf{K})\end{array}$ & $\begin{array}{l}C_{P}^{s} \\
(\mathrm{~K})\end{array}$ & & \\
\hline 154.83 & 1536.2 & 928.1 & 2820.0 & & \\
\hline \multicolumn{2}{|c|}{$\left\langle\left(Y_{1,2}\right)^{2}\right\rangle$} & \multicolumn{2}{|c|}{$\left\langle\left(Y_{3,4,5}\right)^{2}\right\rangle$} & $\left\langle P^{2}\right\rangle$ & \\
\hline $0.116^{\mathrm{a}}$ & $0.096^{\mathrm{b}}$ & $0.058^{\mathrm{a}}$ & $0.068^{\mathrm{b}}$ & $4 \pi$ & \\
\hline
\end{tabular}
which leads to the following values:

TABLE I. Parameters entering the free-energy matrix $\overleftrightarrow{\mathrm{h}}\left(l, l^{\prime} ; T\right)$ [Eqs. (12), (13), and (14)] for $l$ and $l^{\prime}$ far from the surface. (a) $T=160 \mathrm{~K}$, (b) $T=300 \mathrm{~K}$. 
TABLE II. The surface forces conjugate to the variables $x_{3}(1), P_{3}(1)$, and $Y_{1}(1)$ calculated with the microscopic interaction parameter from Ref. 11.

\begin{tabular}{lrrrr}
\hline & $\mathrm{BM}^{\mathrm{a}}$ & \multicolumn{1}{c}{$\mathrm{VW}^{\mathrm{b}}$} & \multicolumn{1}{c}{$\mathrm{C}^{\mathrm{c}}$} & Sum \\
\hline$f_{x_{3}}(\mathrm{~K} / \AA)$ & 11114.2 & -1659.6 & -2358.0 & 7096.7 \\
$f_{P_{3}}(\mathrm{~K})$ & 1295.3 & -178.7 & 187.3 & 1303.9 \\
$f_{Y_{1}}(\mathrm{~K})$ & 2858.7 & -314.6 & -5921.8 & -3404.7 \\
\hline \hline
\end{tabular}

${ }^{\text {aB Born-Mayer. }}$

ban der Waals.

${ }^{\mathrm{c} C o u l o m b .}$

$$
\begin{aligned}
& \bar{J}_{0}^{A}=\frac{9 \pi Q^{2}}{5 \sqrt{2} a^{5}}, \quad J_{1}^{A}=-\frac{39 \pi Q^{2}}{40 \sqrt{2} a^{5}}, \\
& \bar{J}_{0}^{B_{1}}=-\frac{16 \pi Q^{2}}{5 \sqrt{2} a^{5}}, \quad J_{1}^{B_{1}}=\frac{11 \pi Q^{2}}{20 \sqrt{2} a^{5}}, \\
& \bar{J}_{0}^{B_{2}}=\frac{19 \pi Q^{2}}{5 \sqrt{2} a^{5}}, \quad J_{1}^{B_{2}}=-\frac{6 \pi Q^{2}}{5 \sqrt{2} a^{5}}, \\
& \bar{J}_{0}^{E}=-\frac{6 \pi Q^{2}}{5 \sqrt{2} a^{5}}, \quad J_{1}^{E}=\frac{13 \pi Q^{2}}{10 \sqrt{2} a^{5}},
\end{aligned}
$$

where $Q$ is the electric quadrupole constant of the anion.

Because of the long range of the dipole-dipole interactions the dipolar energy is shape dependent. ${ }^{18}$ In the present calculations we have adopted a geometry of a semi-infinite slab, in which the lattice sums have been first taken over the dipoles lying within lattice planes. Such a summation is known to be unambiguous. ${ }^{19}$ The interactions between parallel lattice planes then turn out to decrease with the planes' separation faster than exponentially. ${ }^{20}$ Therefore, the limitation to first neighboring planes implicit in Eqs. (12) and (13) does not introduce a large error.

All the coefficients needed to construct the matrix $\overleftrightarrow{\mathrm{h}}\left(l, l^{\prime}, T\right)$ for $l, l^{\prime}$ far from the surface are collected in Table I. The quadrupole constant $Q$ has been put $Q=0.6 Q_{0}$, where $Q_{0}$ is the quadrupole constant of the free $\mathrm{CN}^{-}$anion. As has been discussed in Ref. 11, the magnitude of the quadrupole moment of the $\mathrm{CN}^{-}$in the bulk (and also at the surface) is not well known. Very likely it depends on the instantaneous orientation of the ion with respect to its crystalline surrounding. The value $Q=0.8 Q_{0}$ used in Ref. 11 seems to be too high compared to other interaction parameters applied here, since it leads to an instability of the lattice at $\mathbf{k} \neq 0$, contrary to the experimental instability at $\mathbf{k}=0$.

The values of the forces $f(l)$ are opposite to the first derivatives of the lattice energy with respect to the variables $x_{3}(l), y_{3}(l), P_{3}(l)$, and $Y_{1}(l)$, the other derivatives being zero by symmetry. By using the microscopic interaction parameters from Ref. 11 and applying the approximations used above, one obtains significant values for $l=1$ only. The microscopic forces being central, the value of $f_{y_{3}}$ vanishes. The remaining forces are gathered in Table II, where contributions from different kinds of interactions are given separately.

\section{STRUCTURE OF THE (001) IDEAL SURFACE OF KCN}

The Green function $\overleftrightarrow{\mathrm{g}}\left(l, l^{\prime} ; T\right)$ [Eqs. (10) and (11)] has the same symmetry properties as the free-energy matrix $\overleftrightarrow{\mathrm{h}}\left(l, l^{\prime} ; T\right)$. Therefore, the variables transforming according to different irreducible representations $A_{1}, B_{1}, B_{2}$, and $E$ of the group $p 4 \mathrm{~mm}$ can be treated independently. To evaluate the Green function for every block we use the surface response theory due to Dobrzynski. ${ }^{6}$ The first step then is the bulk Green function. The explicit formula for the one-dimensional blocks $B_{1}$ and $B_{2}$ is analogous to that used by Mazur and Maradudin ${ }^{21}$ and Zieliński, ${ }^{8}$

$$
\begin{aligned}
& G_{\lambda}\left(l, l^{\prime}\right)=\frac{1}{J_{1}^{\lambda}} \frac{z_{\lambda}^{\left|l-l^{\prime}\right|+1}}{z_{\lambda}^{2}-1}, \\
& \lambda=B_{1}, B_{2},
\end{aligned}
$$

where

$$
z_{\lambda}= \begin{cases}-r_{\lambda}+\left(r_{\lambda}^{2}-1\right)^{1 / 2} & \text { if } r_{\lambda}>1 \\ -r_{\lambda}-\left(r_{\lambda}^{2}-1\right)^{1 / 2} & \text { if } r_{\lambda}<-1\end{cases}
$$

while

$$
r_{\lambda}=J_{0}^{\lambda} / 2 J_{1}^{\lambda}
$$

In the case of the four-dimensional blocks $A_{1}$ and $E$ the formula for the bulk Green function is analogous to that used in Ref. 22. To get it one passes to the Fourier transform

$$
\overleftrightarrow{\mathrm{h}}_{\lambda}(k)=\sum_{\Delta l=-\infty}^{\infty} \overleftrightarrow{\mathrm{h}}_{\lambda}(l, l+\Delta l) e^{i k \Delta l a} .
$$

Replacing $e^{i k \Delta l a}$ by $z$ one obtains $h_{\lambda}(k)=h_{\lambda}(z)$. The Green function $G_{\lambda, \sigma \rho}\left(l, l^{\prime}\right)$ then reads

TABLE III. The parameters $z_{n}$ of formulas (18) and (22) for the (001) surface of KCN at $T=300 \mathrm{~K}$.

\begin{tabular}{rrrrl}
\hline \hline$\lambda$ & $A_{1}$ & $B_{1}$ & $B_{2}$ & \multicolumn{1}{c}{$E$} \\
\hline$z$ & -0.499 & & & 0.023 \\
& 0.207 & -0.268 & 0.061 & $0.250-i 0.309$ \\
& -0.058 & & & $0.250+i 0.309$ \\
\hline \hline
\end{tabular}




$$
G_{\lambda, \sigma \rho}\left(l, l^{\prime}\right)=\frac{1}{H_{\lambda}} \sum_{n=1}^{M} \frac{A_{\lambda, \rho \sigma}\left(z_{n}\right) z_{n}^{l-l^{\prime}+M}}{\left(z_{n}^{2}-1\right) \prod_{\substack{m=1 \\ m \neq n}}^{M}\left(z_{n}-z_{m}\right)\left(z_{n}-z_{m}^{-1}\right)},
$$

where $z_{n}:\left|z_{n}\right| \leq 1$ are the roots of the equation $\operatorname{det}\left[\overleftrightarrow{\mathrm{h}}_{\lambda}(z)\right]=0 . H_{\lambda}$ is the coefficient of the highest power of $z$ in the function $\operatorname{det}\left[\overleftrightarrow{\mathrm{h}}_{\lambda}(z)\right], A_{\lambda, \sigma \rho}$ is the cofactor of the matrix element $h_{\lambda, \rho \sigma}(z)$. For more details of this formula see Ref. 22. In our case $M=3$ for $\lambda=A_{1}$ and $\lambda=E$. The quantities $z_{n}$ which depend on $\lambda$ and on temperature define the rate of the decrease in the distortions caused by a local force with the distance $\left|l-l^{\prime}\right|$. The values of the parameters $z_{n}$ at $T=300 \mathrm{~K}$ obtained with parameters of Table I are given in Table III.

The Green function for the system with surfaces is determined by the bulk Green function and by a cleavage operator $^{6,22}$ which accounts for the changes of the interaction parameters near to the surface. As a first approximation we have assumed that the self-interaction $C_{\lambda}^{s}$ and the orientational susceptibilities are not affected by the surface. The resulting profiles of $x_{3}(l)$ and $y_{3}(l)$ according to formula (10) are shown in Fig. 1 and the

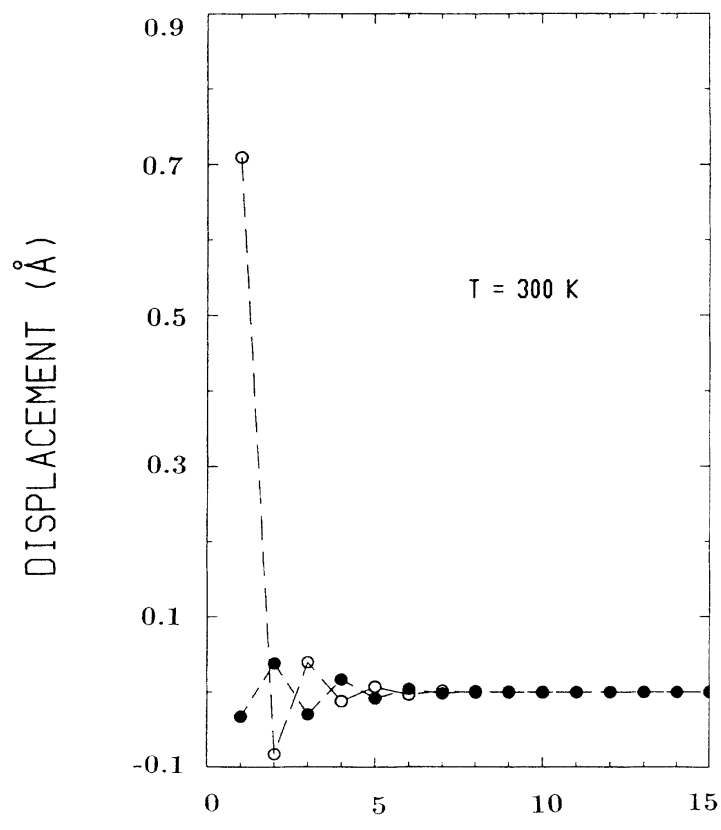

\section{LATTICE PLANE I}

FIG. 1. Perpendicular surface relaxation at the (001) surface of $\mathrm{KCN}$ at $T=300 \mathrm{~K}$. Open circles $(O)$ represent the relative displacement $x_{3}(l)$ [see Eq. (1a)] of the consecutive neighboring lattice planes $(001)$ in the direction perpendicular to the surface. Solid circles (1) represent the relative displacements $y_{3}(l)$ of the cations $K^{+}$with respect to the anions $\mathrm{CN}^{-}$in the direction perpendicular to the surface within the consecutive lattice planes $l$. The lines are to guide the eye.

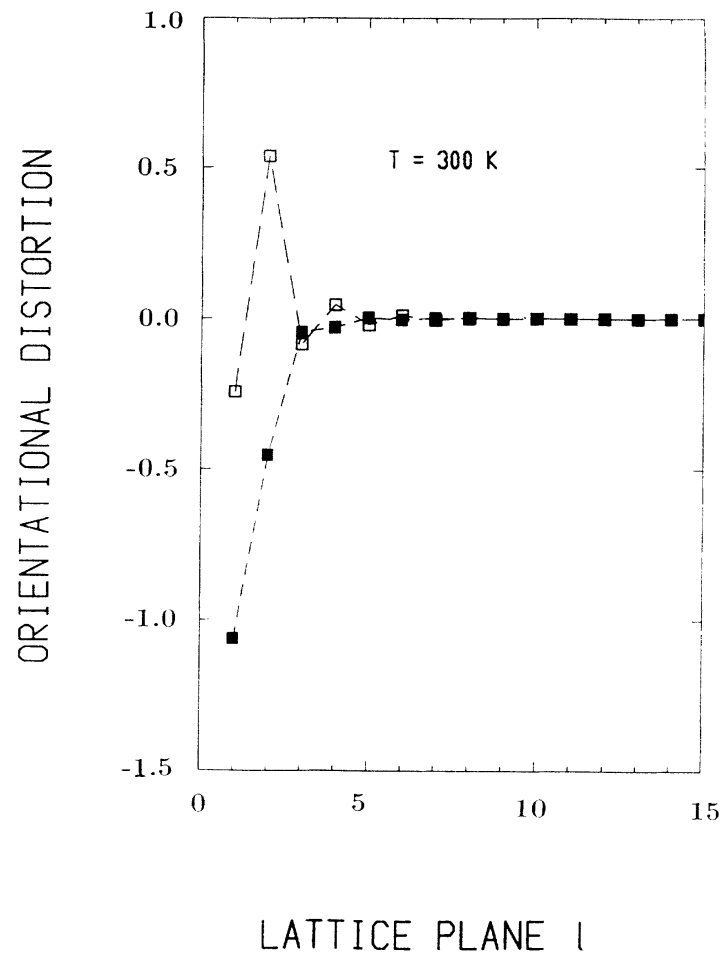

FIG. 2. Profiles of the distortions of the orientational distribution at the (001) surface of $\mathrm{KCN}$ at $T=300 \mathrm{~K}$. Open boxes ( $\square$ ) represent the orientational variable $P_{3}(l)$ [see Eqs. (7a) and $\left(7 a^{\prime}\right)$ ] proportional to the polarization perpendicular to the surface at the consecutive lattice planes $l$. Solid boxes (a) represent the orientational variable $Y_{1}(l)$ [see Eqs. (7b) and $\left(7 b^{\prime}\right)$, which describes the increase in the probability of the orientation of the long axis of the anion $\mathrm{CN}^{-}$in the direction (001).

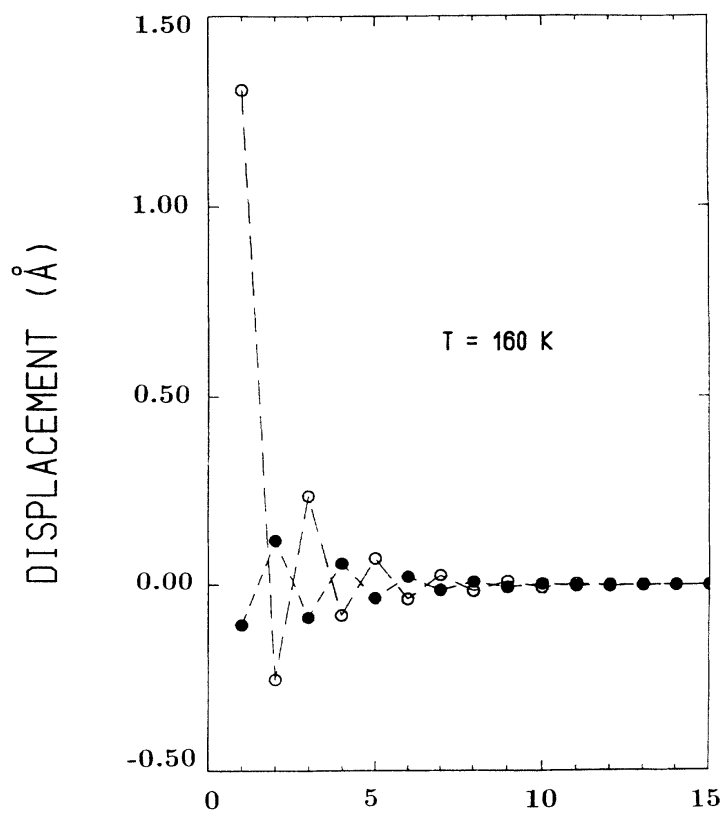

LATTICE PLANE ।

FIG. 3. Same as in Fig. 1 but at $T=160 \mathrm{~K}$. 
profiles of the orientational variables $P_{3}(l)$ and $Y_{1}(l)$ in Fig. 2. In similarity to atomic crystals the predicted surface relaxation of the (001) surface of KCN increases the distance between the two first lattice planes: $x_{3}(1)>0$. Then an oscillating behavior can be seen. This can be called local antiferroelasticity.

The negative value of $Y_{1}(1)$ is the result of the prevailing electrostatic forces between the cations and the negative end charges of the $\mathrm{CN}^{-}$anion, which tend to keep the anion oriented parallel to the surface in opposition to the repulsive Born-Mayer forces (see Table II). The distortion of the orientational distribution at $l=1$ then is an oblate ellipsoid.

The permanent dipoles of the $\mathrm{CN}^{-}$ions have a tendency to align perpendicular to the surface thus transforming the neutral lattice plane into a double electrostatic layer. It is seen from Fig. 2 that the arrangement of the dipoles form a local antiferroelectric structure. This property is to be related to the reactivity of the surface with polar molecules, e.g., $\mathrm{H}_{2} \mathrm{O}$.

To illustrate the influence of the temperature on the structure of the surface the same profiles of $x_{3}(l), y_{3}(l)$, $P_{3}(l)$, and $Y_{1}(l)$ are plotted in Figs. 3 and 4 at $T=160 \mathrm{~K}$, i.e., near to the bulk phase transition. At this temperature $z_{A_{1}}=-0.663, z_{A_{2}}=0.377$, and $z_{A_{3}}=-0.086$.

One can notice an important increase in $x_{3}(1)$ which becomes half as large as the bulk lattice plane separation $a=3.26 \AA$. This indicates that this surface may undergo a first-order relaxation phase transition ${ }^{7}$ within the disordered bulk phase.

Figures 5 and 6 show the influence of an external elec-

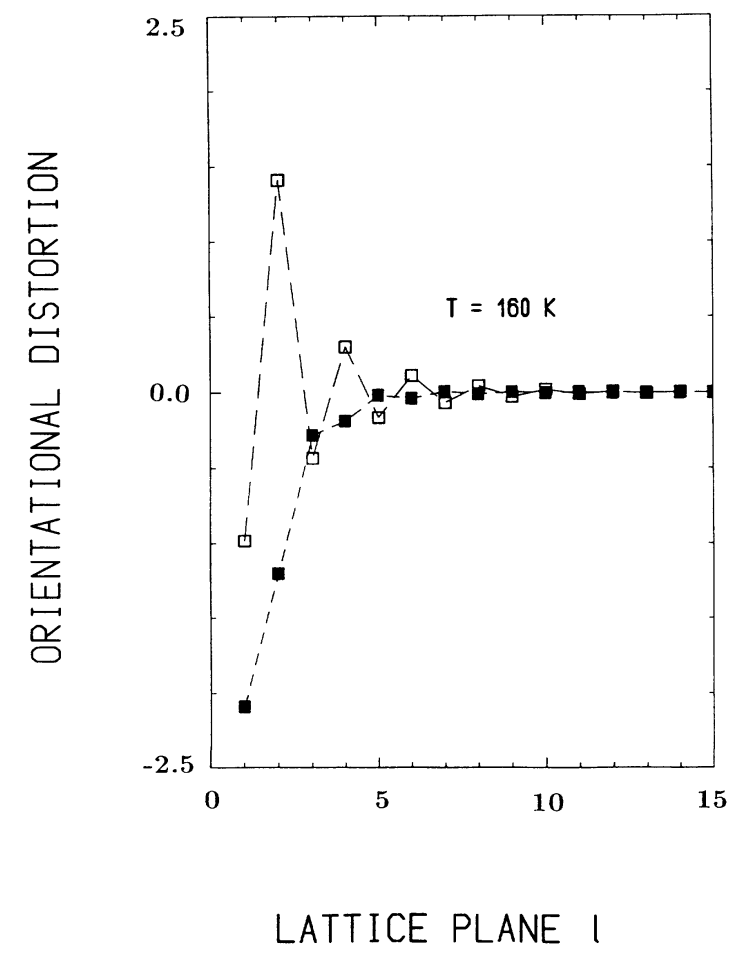

FIG. 4. Same as in Fig. 2 but at $T=160 \mathrm{~K}$.

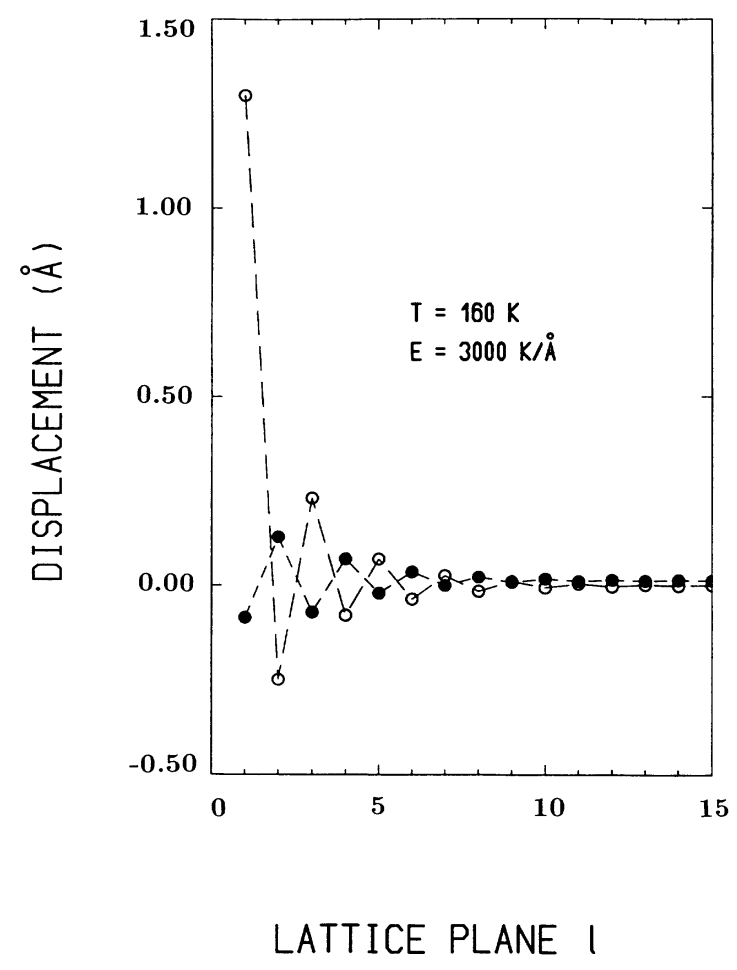

FIG. 5. Same as in Fig. 3 but under the electric field $E=3000$ $\mathrm{K} / \AA ̊$ perpendicular to the surface.

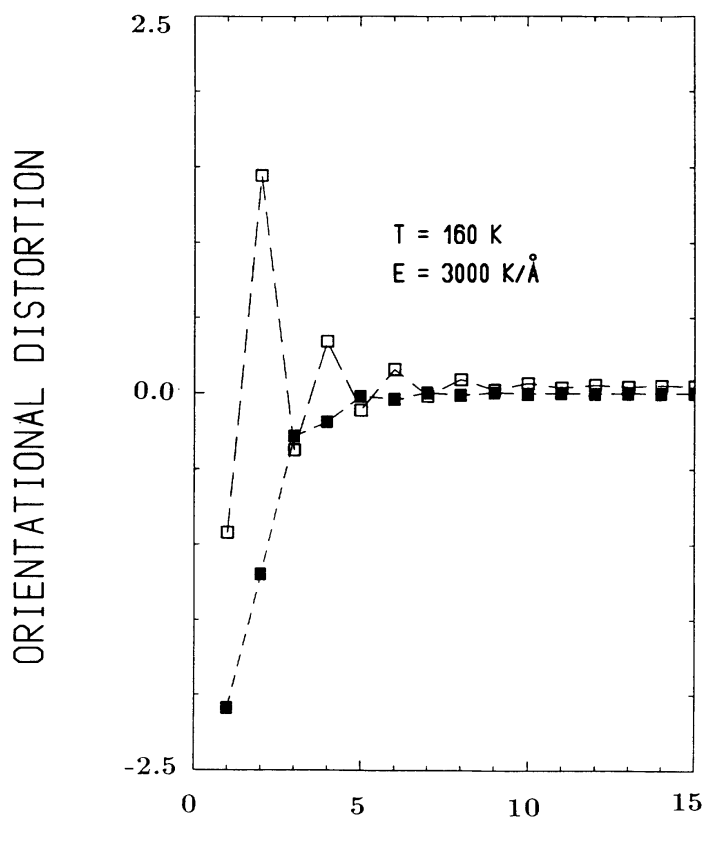

\section{LATTICE PLANE I}

FIG. 6. Same as in Fig. 4 but under the electric field $E=3000$ $\mathrm{K} / \AA ̊$ perpendicular to the surface. 
tric field applied perpendicular to the surface at $T=160$ $K$. The value of the external field has been chosen $E=3000 \mathrm{~K} / \AA$ to be comparable with the local fields acting on the ions (see Table II). The external field which is assumed homogeneous, i.e., $l$ independent, couples linearly to the variable $y_{3}(l)$ and $P_{3}(l)$. The latter coupling is related with the electric dipole of the anion $\mathrm{CN}^{-}$only and not with the steric forces distinguishing its both ends. With the value of the permanent dipole $0.0776 e \AA$ the field coupled to the variable $P(l)$ and produced by the external electric field $E=3000 \mathrm{~K} / \AA$ is $476 \mathrm{~K} / \AA^{2}$.

Since no microscopic forces of symmetry $E$ arise at the ideal (001) surface of $\mathrm{KCN}$ there is no $l$ dependence of the corresponding variables predicted without external influences. Figures 7 and 8 show the profiles of $x_{1}(l)$, $Y_{1}(l), P_{1}(l)$, and $Y_{5}(l)$ under the electric field $E=3000$ $\mathrm{K} / \AA$ parallel to direction $(100)$ at $T=300 \mathrm{~K}$. Having no exact data on the self-interactions $C_{Y}^{s}$ and $C_{P}^{s}$ as well as on the orientational susceptibilities near to the surface we have illustrated the influence of their changes by making $h_{E, 33}(1,1)$ and $h_{E, 44}(1,1) 20 \%$ lower than the corresponding matrix elements in the bulk. The resulting profiles are given in Figs. 9 and 10. It should be noted that the changes of the interaction parameters near the surface may lead to a surface reconstruction phase transition.

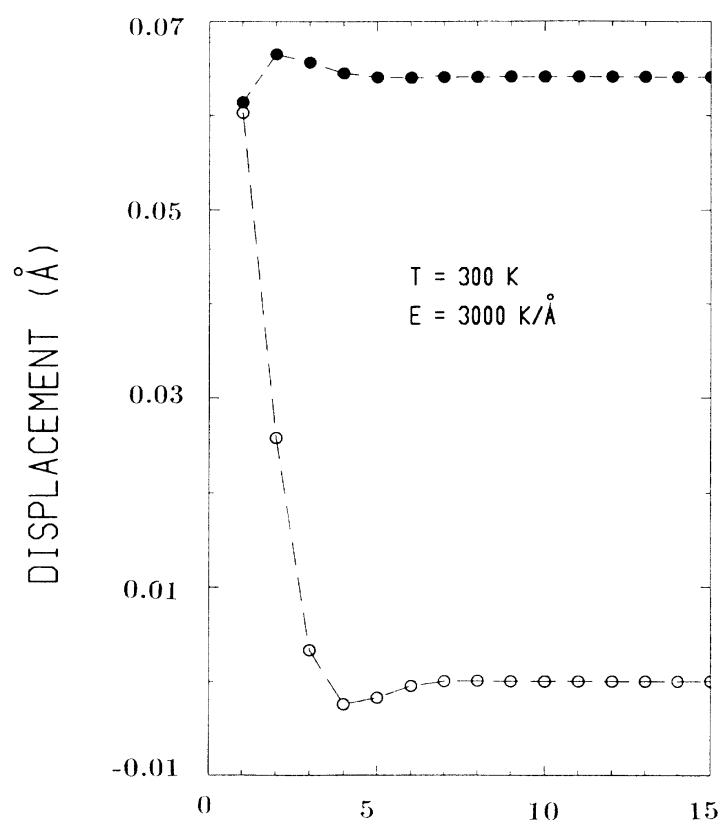

\section{LATTICE PLANE ।}

FIG. 7. Parallel surface relaxation at the (001) surface of $\mathrm{KCN}$ at $T=300 \mathrm{~K}$ and under electric field $E=3000 \mathrm{~K} / \AA$ in the direction (100). Open circles $(O)$ represent the relative displacements $x_{1}(l)$ [see Eq. (1a)] of the consecutive neighboring lattice planes $l$ in the direction (100) parallel to the surface. Solid circles $(\bigcirc)$ represent the relative displacement $y_{1}(l)$ [see Eq. (1b)] of the cation $\mathrm{K}^{+}$with respect to the anion $\mathrm{CN}^{-}$in the direction (100) within the consecutive lattice planes $l$.

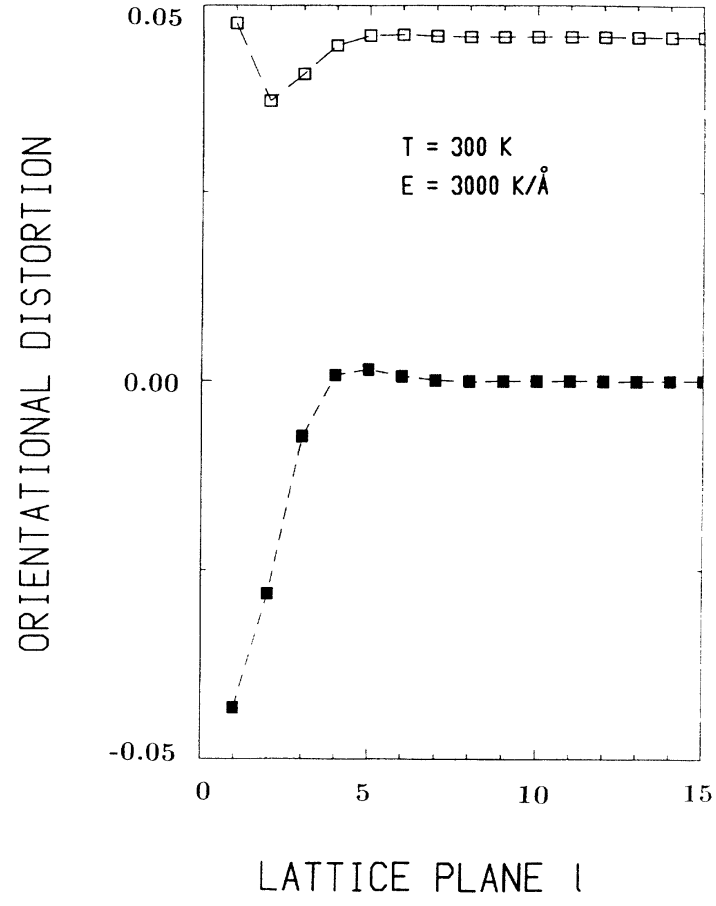

FIG. 8. Profiles of the orientational variables $P_{1}(l)$ and $Y_{5}(l)$ at the (001) surface of $\mathrm{KCN}$ at $T=300 \mathrm{~K}$ and under electric field $E=3000 \mathrm{~K} / \AA$ in the direction (100). Open boxes ( $\square$ ) represent the orientational variable $P_{1}(l)$ [see Eqs. (7a) and (7a')] proportional to the polarization in the direction (100) parallel to the surface within consecutive lattice planes $l$. Solid boxes ( $\boldsymbol{\square})$ represent the orientational variable $Y_{5}(l)$ [see Eqs. (7c) and $\left(7 c^{\prime}\right)$ ] which describes the increase in the orientation probability in the direction (011) for the consecutive lattice planes $l$.

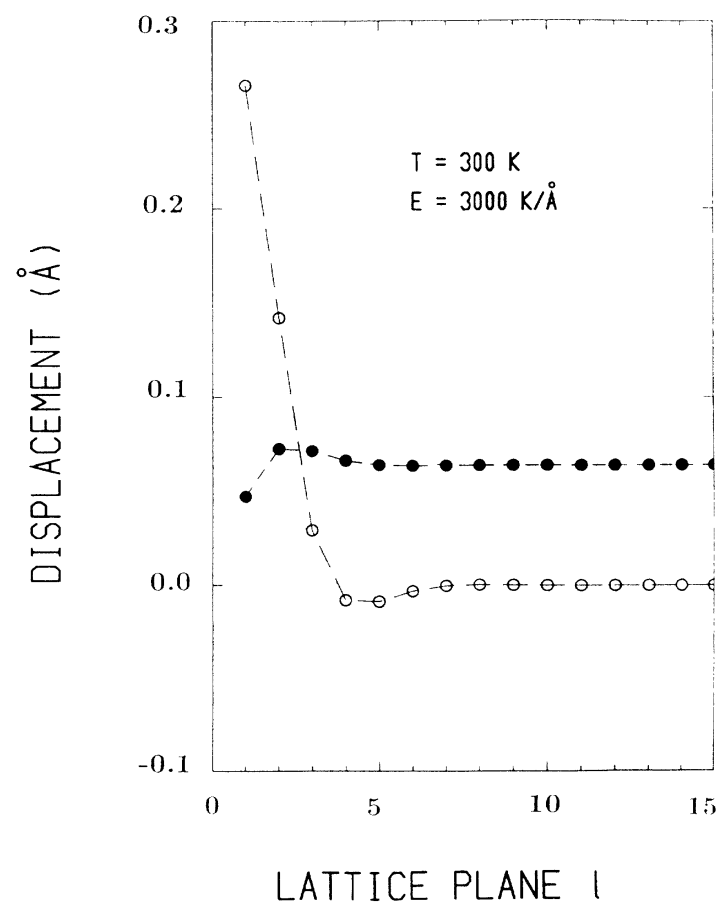

FIG. 9. Same as in Fig. 7 but with the surface matrix elements $h_{E, 33}(1,1)$ and $h_{E, 44}(1,1)$ [see Eq. (13a)] $20 \%$ smaller than in the bulk. 


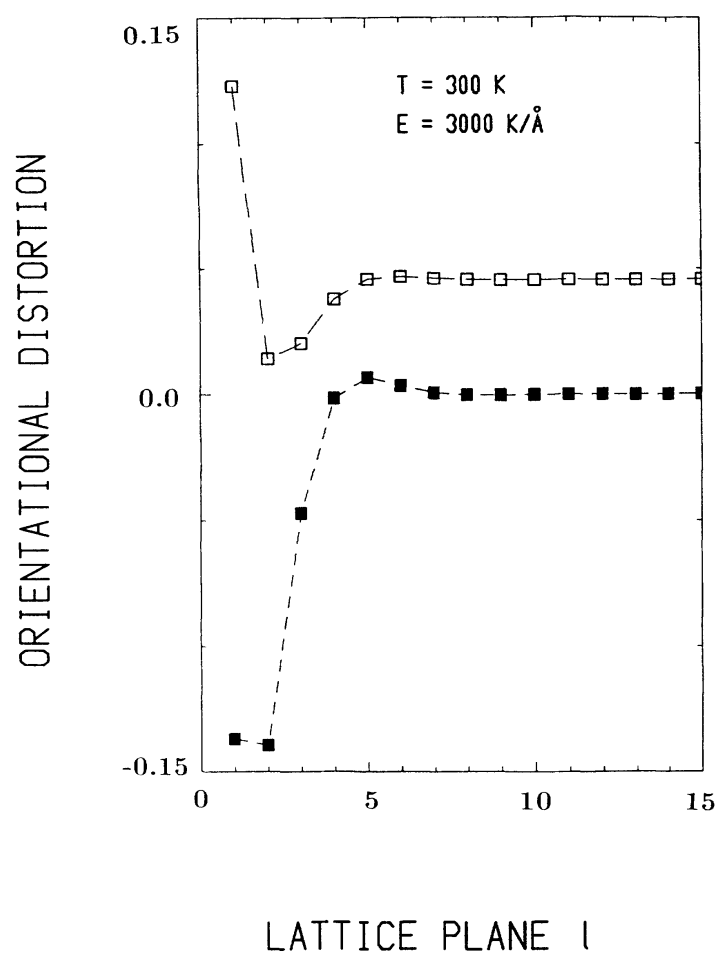

FIG. 10. Same as in Fig. 8 but with the surface matrix elements $h_{E, 33}(1,1)$ and $h_{E, 44}(1,1)$ [see Eq. (13a)] 20\% smaller than in the bulk.

\section{DISCUSSION}

The above analysis is an attempt to predict theoretically the structure and the orientational disorder of an ideal surface of the orientationally disordered crystal of $\mathrm{KCN}$ on the basis of the microscopic parameters determined by experiments and model calculations for the bulk material. Whereas such analyses are known for ordered crystals with the use of the lattice energy calculations ${ }^{3}$ the present theory requires rather the lattice free energy [Eq. (8)]. The neglect of the higher-order terms in $\mathbf{w}(l)$ in Eq. (8) makes the present analysis valuable for unreconstructed surface under relatively small forces $\mathbf{f}(l)$. In the case of strongly reconstructed surfaces only the asymptotic behavior of the variables $\mathbf{w}(l)$ relatively far from the surface is predicted correctly and determined by the parameters $z$ from the Table III. No instabilities leading to a reconstruction have been obtained here, but this is liable to be changed when more exact data on the self-interactions and the one-particle susceptibilities of the surface anions were known. The experimental data on the real structure of the (001) surface of the cubic $\mathrm{KCN}$ are not known for the moment so that an immediate comparison with experiment cannot be made. The present results are, nevertheless, also interesting as a basic step towards the crystal growth theory and the theory of chemical reactivity of the surface.

\section{ACKNOWLEDGMENTS}

This work has been supported by Austrian "Ministerium für Wissenschaft und Forschung." A part of it has been done during the stay of one of the authors (P.Z.) at the Universitaire Instelling Antwerpen. The authors thank Professor H. Warhanek for his hospitality during their stay in Vienna. P.Z. thanks L. Dobrzynski (D. R. at CNRS) for discussion and advice. The assistance of $A$. Majerowicz in work with computer facilities is thankfully acknowledged.
*Permanent address: Institute of Nuclear Physics, ul. Radzikowskiego 152, 31-342 Kraków, Poland.

${ }^{1}$ See, for example, The Structure of Surfaces II, edited by J. F. van der Veen and M. A. van Hove (Springer, Berlin, 1988); Chemistry and Physics of Solid Surfaces VIII, edited by R. Vanselow and R. Howe (Springer, Berlin, 1990).

2J. F. Scott, H. M. Duiker, P. D. Beale, B. Pouligny, K. Dimmler, M. Parris, D. Butler, and S. Eaton, Physica B 150, 160 (1988), and references given therein.

${ }^{3}$ K. Kobashi and R. Etters, J. Chem. Phys. 67, 4341 (1985).

${ }^{4} \mathrm{G}$. Benedek, Surf. Sci. 61, 604 (1976).

${ }^{5}$ F. Zandiehnadem and W. Y. Ching, Phys. Rev. B 41, 12162 (1990).

${ }^{6}$ L. Dobrzynski, Surf. Sci. Rep. 6, 119 (1986).

${ }^{7}$ P. Zieliński, Phys. Rev. B 38, 12338 (1988).

${ }^{8}$ P. Zieliński, J. Phys. Condens. Matter 2, 857 (1990).

${ }^{9}$ D. Sahu and S. D. Mahanti, Phys. Rev. B 26, 2981 (1982); 29, 340 (1984).

${ }^{10}$ K. H. Michel and J. M. Rowe, Phys. Rev. B 32, 5818 (1985).

${ }^{11}$ K. H. Michel and J. M. Rowe, Phys. Rev. B 32, 5827 (1985).
${ }^{12}$ K. H. Michel and J. Naudts, J. Chem. Phys. 67, 547 (1977).

${ }^{13}$ K. H. Michel and K. Parlinski, Phys. Rev. B 31, 1823 (1985).

${ }^{14}$ D. M. Kroll and R. Lipowsky, Phys. Rev. B 28, 6435 (1983); D. M. Kroll and G. Grompper, ibid. 36, 7078 (1987).

${ }^{15}$ D. R. Tilley and B. Žekš, Solid State Commun. 49, 3885 (1984).

${ }^{16}$ International Tables for Crystallography, edited by T. Hahn (Reidel, Dordrecht, 1987), Vol. A.

${ }^{17}$ D. Strauch, U. Schröder, and W. Bauerfeind, Solid State Commun. 30, 559 (1979).

${ }^{18}$ M. F. Thorpe and S. W. de Leeuw, Phys. Rev. B 33, 8490 (1986).

${ }^{19}$ P. Zieliński and K. Żabińska, Acta Phys. Pol. A 74, 479 (1988).

${ }^{20}$ A. A. Maradudin, E. W. Montroll, G. H. Weiss, and I. R. Ipatowa, Theory of the Lattice Dynamics in the Harmonic Approximation (Academic, New York, 1971), p. 541.

${ }^{21}$ P. Mazur and A. A. Maradudin, Phys. Rev. B 24, 2996 (1981).

${ }^{22}$ P. Zieliński and L. Dobrzynski, Phys. Rev. B 41, 10377 (1990). 